\title{
Survivin selective inhibitor YM155 promotes cisplatin-induced apoptosis in embryonal rhabdomyosarcoma
}

\author{
TAKEHISA UENO, SHUICHIRO UEHARA, KENGO NAKAHATA and HIROOMI OKUYAMA \\ Pediatric Surgery, Osaka University Graduate School of Medicine, Suita, Osaka 565-0871, Japan
}

Received January 22, 2016; Accepted February 26, 2016

DOI: $10.3892 / \mathrm{ijo} .2016 .3438$

\begin{abstract}
Survivin, a member of the inhibitor of apoptosis protein family, functions as a key regulator of programmed cell death. YM155 is a small molecule that selectively inhibits survivin. We investigated the effect of YM155 on survivin suppression in the human rhabdomyosarcoma (RMS) cell line RD. The efficacy of YM155 in combination with cisplatin was also determined in a xenograft model. The effect of YM155 on survivin expression in the RD cell line was examined at both mRNA and protein levels using real-time PCR and western blot analysis. RD cells were cultured with various concentrations of YM155, then cisplatin was added to the medium and the anti-proliferation response was determined. Cell growth was evaluated by WST-8 assay. Finally, the efficacy of YM155 combined with cisplatin was examined in an established xenograft model. Survivin mRNA levels in the RD cell line were decreased to 72 and $24 \%$ at 24 and 48 h, respectively, after $10 \mathrm{nM}$ of YM155 was added. YM155 also decreased the levels of survivin protein. YM155 treatment (10 nM) inhibited cell proliferation of RD in a dose-dependent manner in vitro, with $58 \%$ of cells viable at $48 \mathrm{~h}$. When cultured with $10 \mathrm{nM}$ of YM155 and $10 \mu \mathrm{M}$ cisplatin, RD cells demonstrated only $25 \%$ of the growth observed when cultured with cisplatin alone. YM155 in combination with cisplatin significantly inhibited tumor growth by $13 \%$ compared with control $(\mathrm{P}<0.0001)$ in RD xenograft tumors. YM155 increased the sensitivity of cisplatin by suppressing survivin in the embryonal RMS cell line RD. Further studies should investigate the use of YM155 as an apoptosis inducer, either alone or in combination with cisplatin, for the treatment of malignant RMS.
\end{abstract}

Correspondence to: Dr Takehisa Ueno, Department of Pediatric Surgery, Osaka University Graduate School of Medicine, 2-2 Yamadaoka, Suita, Osaka 565-0871, Japan

E-mail: ueno@pedsurg.med.osaka-u.ac.jp

Abbreviations: RMS, rhabdomyosarcoma; PCR, polymerase chain reaction; FACS, fluorescence activated cell sorting; TUNEL, terminal deoxynucleotidyl transferase dUTP-biotin nick end-labeling

Key words: rhabdomyosarcoma, survivin, YM155, cisplatin, RD, apoptosis, xenotransplant

\section{Introduction}

Rhabdomyosarcoma (RMS) is the most common soft tissue sarcoma in children, the incidence of RMS in children $<20$ years old is 4.3 per million per year (1). Current treatment for RMS relies on chemotherapy, along with surgery and radiotherapy. The prognosis for non-metastatic RMS has improved considerably over the last 30-40 years due to use of combination chemotherapy (2-4). Despite significant improvements in survival over the past few decades, more than one-third of RMS patients continue to die of the disease (5). In particular, the outcomes of patients with metastatic disease remain very poor, despite intensification of treatment with agents known to be active and attempts at introducing novel therapies (6-8). The mainstay of chemotherapy for RMS continues to be vincristine and actinomycin $\mathrm{D}$ in combination with cyclophosphamide, so-called VAC treatment. The most recent Children's Oncology Group (COG) trial for low-risk RMS showed that patients have an excellent outcome [2-year event-free survival (EFS), 88\%; overall survival (OS), 98\%] with short therapy duration (9). Whereas the most recent COG high-risk RMS study showed poor outcome in 3-year EFS 38\% (10).

Other drugs such as doxorubicin, etoposide, and cisplatin have been added to this combination in higher risk patients, but to date there is no definitive proof of improved outcomes with the addition of these drugs, despite evidence of response in the relapse setting (6).

Survivin is a $16.5-\mathrm{kDa}$ member of the inhibitor of apoptosis protein family, and blocks the mitochondrial pathway of apoptosis by inhibiting caspases (11). Survivin is not expressed in differentiated normal tissue $(12,13)$. Higher survivin expression has been correlated with unfavorable survival or disease recurrence in various adult malignancies $(12,14-22)$. In the pediatric setting, most primary hepatoblastoma tissue specimens express survivin, and its expression was shown to increase after chemotherapy, suggesting that survivin may play a role in the survival of tumor cells (23). Survivin has therefore become a therapeutic target of pediatric tumors.

YM155 is a novel, small, imidazolium-based compound that specifically inhibits survivin, as determined using a survivin promoter activity assay (24). In pre-clinical experiments, YM155 as a single agent downregulated the transcription of survivin in a dose/time-dependent manner, triggering p53-independent apoptosis in a wide range of 
human tumor cells. YM155 also induced tumor regression in established cancer xenografts $(24,25)$, and the combination of YM155 with various chemotherapeutic agents potentiated apoptosis induction in several human cancers (26-28). Despite its demonstrated efficacy in targeting tumor cells, the effects of YM155 in combination with DNA-damaging drugs have remained largely unknown.

Once primary chemotherapy fails, cancer cells became resistance to the drug. We used a cell line that was treated by cyclophosphamide, therefore other DNA-damaging drugs should be explored (29).

The platinum compound cisplatin, DNA damaging drug, showed efficacy combined with YM155 in previous reports $(28,30,31)$. In this study, we selected cisplatin combined with YM155 to search for a new regimen. We examined the effects of YM155 in combination with the platinum compound cisplatin on a human RMS cell line both in vitro and in vivo. Given the importance of survivin in cisplatin apoptosis resistance, and with the aim of finding new strategies to reduce cisplatin doses in patients with RMS, we sought to determine whether YM155 synergized with cisplatin to affect the RMS cell line in vitro, and as a tumor xenograft.

\section{Materials and methods}

Cell culture. Cells from the human rhabdomyosarcoma cell line RD were obtained from Japanese Collection of Research Bioresources Cell Bank. Cells were maintained in Gibco RPMI-1640 (Thermo Fisher Scienrific, Waltham, MA, USA), supplemented with $10 \%$ heat-inactivated fetal bovine serum in a humidified incubator with $5 \% \mathrm{CO}_{2}$ at $37^{\circ} \mathrm{C}$.

Reagents. YM155 was purchased from Selleck Chemicals (Houston, TX, USA) and dissolved with DMSA to a final stock solution concentration of $1 \mathrm{mM}$ cisplatin (Wako Pure Chemical Industries, Osaka, Japan) was dissolved in sterile water to a final stock solution concentration of $1 \mathrm{mM}$.

Real-time PCR. RD cells were cultured in medium with or without various concentrations of YM155 and/or cisplatin. Control cultures received dimethyl sulfoxide $0.1 \%$. For RNA extraction, NucleoSpin RNA (Takara, Osaka, Japan) was used according to the manufacturer's protocol. Primix Taq (Takara) was used for the reaction. The primers to detect human Survivin (Invitrogen, Carlsbad, CA, USA) used for PCR were as follows: 5'-GGCTCTTTCTCTGTCCAGTT-3' (forward), 5'-ACCACCGCATCTCTACATTC-3' (reverse). Equal volumes of PCR products were electrophoresed through agarose gel in TBE buffer.

Quantitative PCR. Custom primers to detect human survivin and glyceraldehyde 3-phosphate dehydrogenase (GAPDH) were created by Invitrogen. Sybergreen analysis was carried out with SYBR Premix Ex Taq II (Takara). Quantitative PCR was performed using an Applied Biosystems ViiA 7 System (Thermo Fisher). Results from each sample were compared using the comparative CT method (Applied Biosystems). Genomic levels and cDNA expression levels were measured relative to GAPDH. Experiments were performed in triplicate.
Western blot analysis. Protein was extracted by western blot assays. Extracted protein samples were resolved by SDS-PAGE and transferred to a polyvinylidene difluoride membrane. After blocking at room temperature with TBS-T buffer, each membrane was incubated for $1 \mathrm{~h}$ at $4^{\circ} \mathrm{C}$ with the primary antibodies anti-survivin (Cell Signaling, Beverly, MA, USA) and mouse anti- $\beta$-actin (Cell Signaling). The membranes were then washed with TBS-T and incubated with horseradish peroxidase-conjugated secondary antibody for $1 \mathrm{~h}$ at room temperature. Immunostar Zeta (Wako Pure Chemical Industries) was used as luminescence solution. Proteins of interest were visualized using the ImageQuant LAS 4000 Mini (General Electric Co., Fairfield, CT, USA).

RNA interference. Validated human anti-survivin siRNA was purchased from Life Technologies Corp. (Carlsbad, CA, USA). A control scramble (nonsilencing) siRNA was purchased from Cell Signaling Technology. Cells were seeded at $24 \mathrm{~h}$ before transfection with either anti-survivin siRNA or control scramble siRNA using the Lipofectamine RNAiMAX Transfection reagent (Life Technologies Corp.). The final concentration of siRNA duplex in the culture medium was $50 \mathrm{nmol} / \mathrm{l}$.

Cell growth inhibition assay. The RD cell lines were used to determine the inhibitory effect of YM155 on cell growth by the WST assay, which uses the highly water-soluble tetrazolium salt WST-8. Cells $\left(6 \times 10^{4} /\right.$ well) were plated in 96 -well plates and then cultured in medium with or without various concentrations of YM155 and/or cisplatin. Control cultures received $0.1 \%$ DMSO. At 24 and $48 \mathrm{~h}$, after the treatment, the percentage of viable cells in each well was examined with a Cell Counting Kit-8 (Dojindo Molecular Technologies, Kumamoto, Japan) using a Multiscan Go microplate multispectrophotometer (Thermo Fisher Scientific).

Annexin $V$ assays. Annexin V/propidium iodide staining was carried out using a BD Annexin-V kit (BD Biosciences, Franklin Lakes, NJ, USA), and analyzed by FACS in a BD FACS Canto II flow cytometer (BD Biosciences).

Assay of caspase-3 activity. Sixty thousand cells from each experimental condition were assayed for caspase-3 activity using the Apo-ONE Homogeneous Caspase-3/7 assay (Promega, Madison, WI, USA). This system uses the profluorescent substrate rhodamine 110 bis-(N-CBZL-aspartyl-L-glutamylL-valyl-L-aspartic acid amide; Z-DEVD-R110), which allows highly sensitive, quantitative measurement of caspase-3 protease activity. Caspase activity, measured with the SH-9000 microplate reader (Corona Electronic, Hitachinaka, Japan), is indicated by intense rhodamine 110 fluorescence at $499 \mathrm{~nm}$. The emission maximum is $521 \mathrm{~nm}$. Experiments were performed in triplicate.

In vivo antitumor activities in an RD xenograft model. Four-week-old male mice (NOD SCID) were purchased from Oriental Yeast (Tokyo, Japan). RD cells $\left(2 \times 10^{6}\right)$ were injected into the backs of the mice. After tumors grew to $>20 \mathrm{~mm}$ in diameter, 5-mm cubic sections of tumor were transplanted into the flank of 4-week-old male nude mice (BALB/c nu/nu), 
A

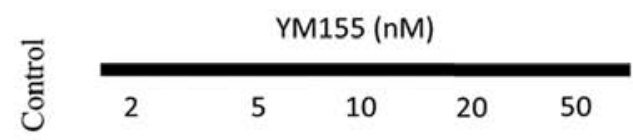

Survivin

GAPDH

C

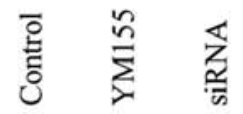

$\mathrm{kDa}$

16.5

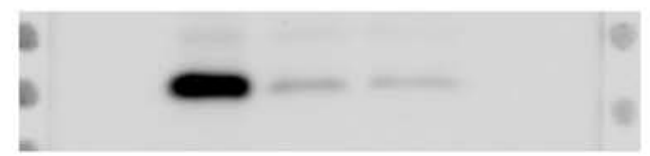

Survivin

45

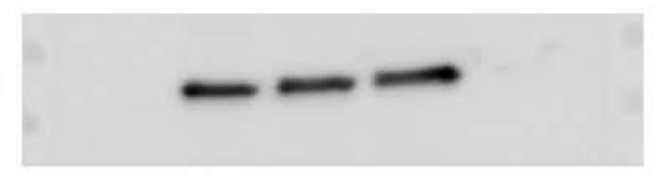

B

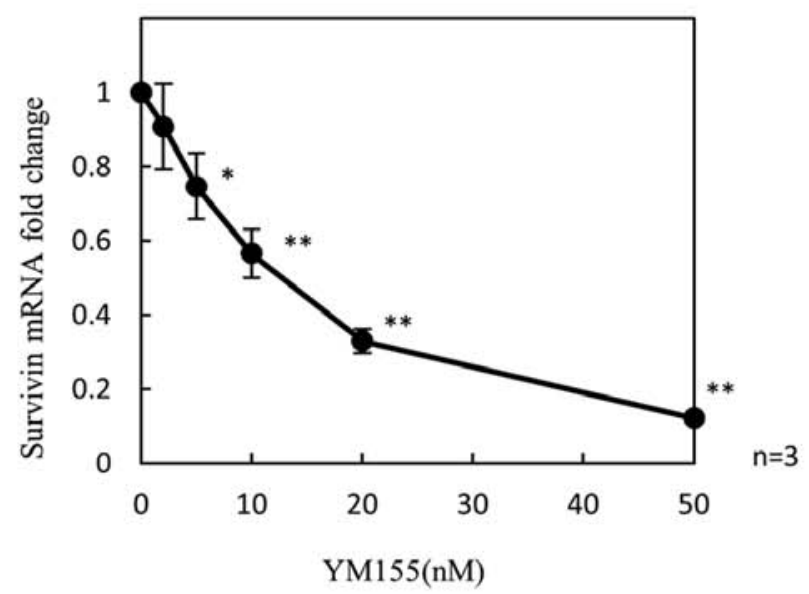

Figure 1. YM155 suppresses survivin expression in human RD cells. Survivin expression decreased after $24 \mathrm{~h}$ of treatment with $2-50 \mathrm{nM}$ of YM155 in a dosedependent manner. (A) Survivin mRNA expression was measured by quantative real-time PCR. YM155 doses of $5 \mathrm{nM}$ and greater suppress survivin mRNA compared to YM155 negative control $\left({ }^{*} \mathrm{P}<0.005,{ }^{* *} \mathrm{P}<0.001\right)$. (B) YM155 suppresses survivin protein in human RD cells as well as siRNA of survivin $(\mathrm{C})$.

purchased from Oriental Yeast. After tumor diameter reached $8 \mathrm{~mm}, 5 \mathrm{mg} / \mathrm{kg}$ of YM155 was administered intraperitoneally (i.p.) once a day for 4 weeks. Cisplatin was administered i.p. at a dose of $2.5 \mathrm{mg} / \mathrm{kg}$ twice a week. Tumor diameters were measured once a week, and tumor volumes were determined (length $\mathrm{x}$ width ${ }^{2}$ )/2. Observations continued until day 28 after transplantation. The experiments were reviewed and approved by the Animal Experimentation Committee of Osaka University (permit no. 25-058-000), and conducted in accordance with institutional guidelines. All efforts were made to minimize suffering.

Immunochemistry. Tumors were harvested at the end of the experiments (after 28 days) as fixed, paraffin wax-embedded sections. Sections were subsequently dewaxed and rehydrated, and survivin expression was determined by staining with survivin, desmin, and myoD antibodies with the Real Envision detection system (Dako Denmark A/S, Glostrup, Denmark). After specific staining or hematoxylin and eosin staining, sections were dehydrated in alcohol and xylene and subsequently mounted on a slide.

Apoptosis stain. Tumor sections were subjected to terminal deoxynucleotidyl transferase dUTP-biotin nick end-labeling (TUNEL) staining, performed with an In Situ Apoptosis Detection kit (Takara) using anti-FITC horseradish peroxidase conjugate.

Statistical analysis. Results are expressed as relative change compared with DMSO controls and are given as the mean \pm SD. The statistical differences between groups were tested using a two-tailed Student's t-test. Statistical significance was assumed for P-values $<0.05$.

\section{Results}

YM155 inhibits endogenous survivin expression in RD cells and induces RMS cell apoptosis in vitro. Endogenous survivin mRNA expression in RD, an RMS cell line, was significantly suppressed in a dose-dependent manner by >10 nM YM155 in vitro, measured by quantitative PCR (Fig. 1A). YM155 suppressed survivin expression at concentrations of $5 \mathrm{nM}$ and higher (Fig. 1B). These results suggested that the suppression of survivin by YM155 occurs via the transcriptional inhibition of the survivin gene. We also used western blotting to confirm that YM155 suppressed survivin protein synthesis as well as siRNA did (Fig. 1C).

To evaluate the ability of YM155 to induce cell death in RD cells, we treated the cells with YM155 for 24 and 48 h, after which cell viability was assessed with the WST-8 method. YM155 concentrations from 1 to $50 \mathrm{nM}$ significantly decreased the viability of cells in a dose-dependent manner (Fig. 2).

Suppressing survivin mRNA expression by siRNA or YM155 enhances cisplatin-induced apoptosis in vitro. RD cells were treated with cisplatin or the combination of siRNA and cisplatin, and cell viability was then assessed with the WST-8 method to assess the in vitro effects on survivin mRNA suppression. Suppressing survivin mRNA with siRNA significantly decreased the viability of cells, suggesting enhancement of cisplatin-induced apoptosis in vitro (Fig. 3). 

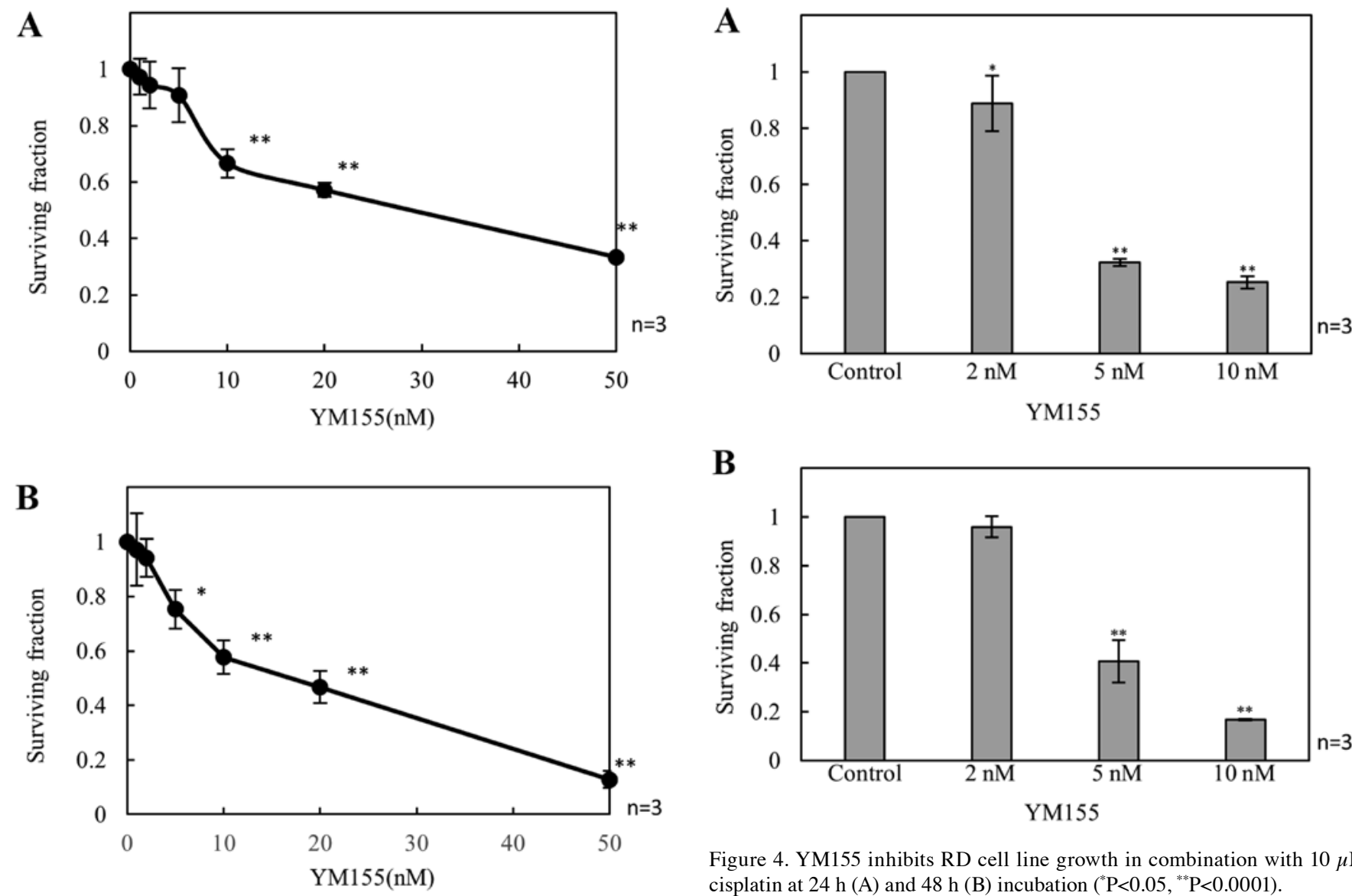

Figure 4. YM155 inhibits RD cell line growth in combination with $10 \mu \mathrm{M}$ cisplatin at $24 \mathrm{~h}(\mathrm{~A})$ and $48 \mathrm{~h}(\mathrm{~B})$ incubation $\left({ }^{*} \mathrm{P}<0.05,{ }^{* *} \mathrm{P}<0.0001\right)$.

Figure 2. YM155 inhibits RD cell line growth at $24 \mathrm{~h}$ (A) and $48 \mathrm{~h}$ (B) incubation in a dose-dependent manner $\left({ }^{*} \mathrm{P}<0.001,{ }^{* *} \mathrm{P}<0.0001\right)$.

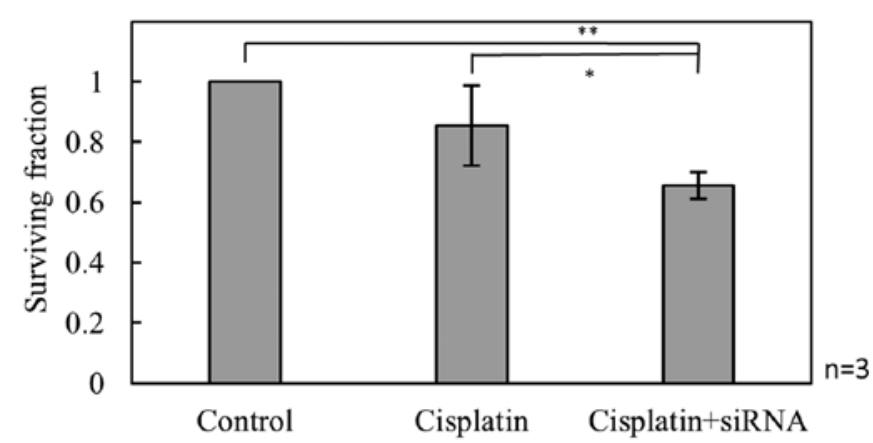

Figure 3. Anti-survivin siRNA inhibits RD cell line growth with $10 \mu \mathrm{M}$ cisplatin at 24-h incubation $\left({ }^{*} \mathrm{P}=0.02,{ }^{* * *} \mathrm{P}=0.0002\right)$.

RD cells were treated with either YM155, cisplatin, or both, after which cell viability was assessed with the WST-8 method to assess the in vitro effects on apoptosis induction. At concentrations from 1 to $50 \mathrm{nM}$, cell viability was significantly decreased in a dose-dependent manner (Fig. 4).

YM155 induces cancer cell apoptosis in vitro. RD cells treated with either YM155, cisplatin, or both were analyzed by Annexin $\mathrm{V}$ assay to assess the in vitro effects on apoptosis induction (Fig. 5). The number of apoptotic cells was increased by $1.2 \%$ by exposure to $10 \mu \mathrm{M}$ cisplatin (Fig. 5B) and $1.3 \%$ by treatment with $5 \mathrm{nM} \mathrm{YM155} \mathrm{(Fig.} \mathrm{5C).} \mathrm{In} \mathrm{contrast,} \mathrm{the}$ combination of cisplatin and YM155 increased the number of apoptotic cells more markedly, by $2 \%$ (Fig. 5D). We next examined the effect of YM155 on cisplatin-induced apoptosis in RD cells using the caspase- 3 assay. Again, combined treatment of RD cells with YM155 and cisplatin induced a synergistic increase in caspase-3 activity (Fig. 6). These data suggest that YM155 promoted the induction of apoptosis caused by a DNA-damaging agent in the RD cell line.

Antitumor effects of YM155 plus cisplatin in the RD cell xenograft model and immunostaining. We evaluated the in vivo antitumor activity of YM155 with i.p. injection into mice xenografted subcutaneously with RD cells as shown in Fig. 7. Mice receiving daily i.p. bolus injections of YM155 at $5.0 \mathrm{mg} / \mathrm{kg}$ showed a maximum tumor growth inhibition of $31 \%$ compared with control. YM155 showed time-dependent antitumor activity in vivo. Mice receiving twice-a-week i.p. injections of cisplatin showed a maximum tumor growth inhibition of $46 \%$ compared with control. YM155 in combination with cisplatin significantly inhibited tumor growth by $13 \%$ compared with control $(\mathrm{P}<0.0001)$. However, each monotherapy suppressed tumor growth, which was followed by an acceleration of growth during the observation period. These results indicate that YM155 in combination with cisplatin was tolerated in mice and enhanced the in vivo tumor response to cisplatin (Fig. 7).

Xenograft tumors in all treatment groups were visualized by microscopy in vivo, and then resected. Confirming that the 
A

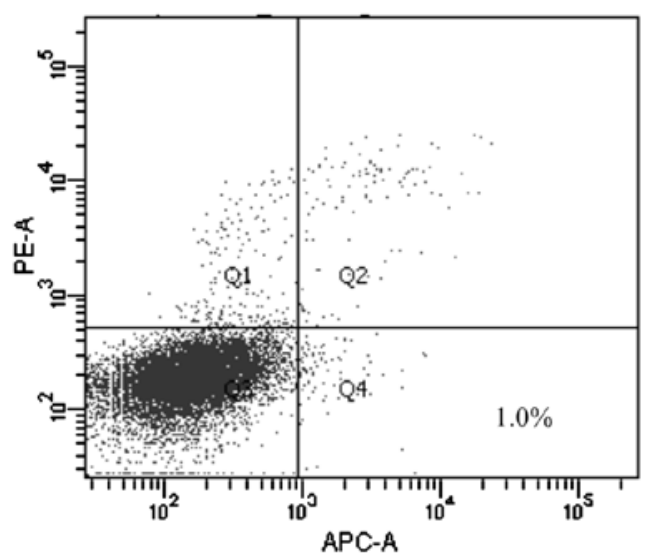

C

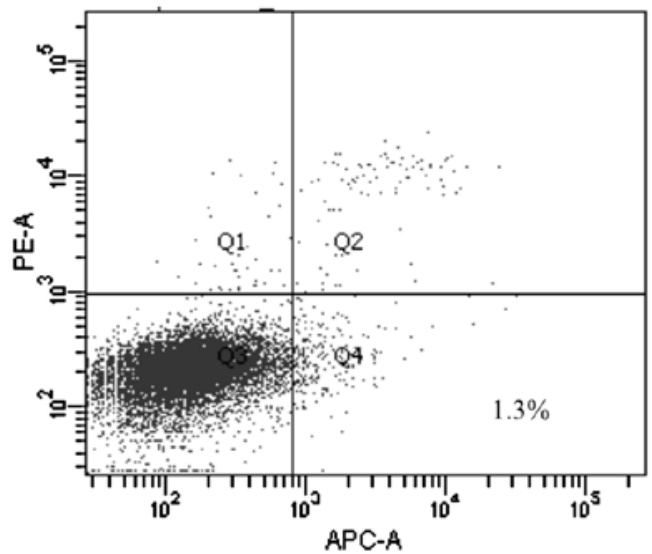

B

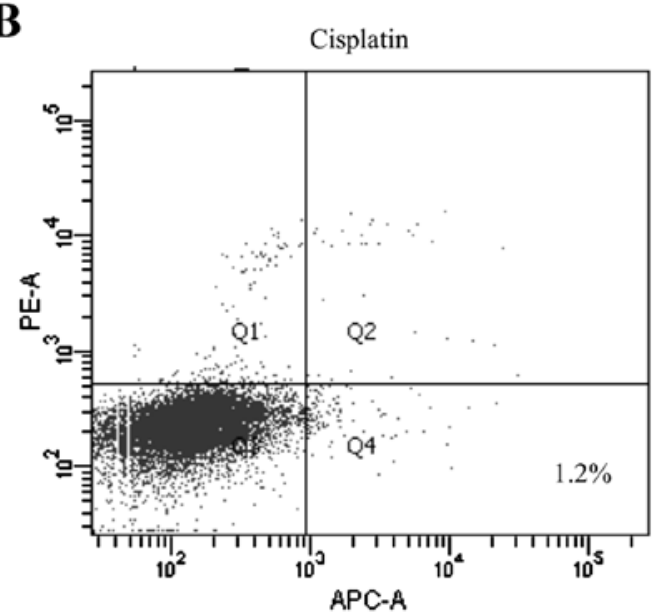

D

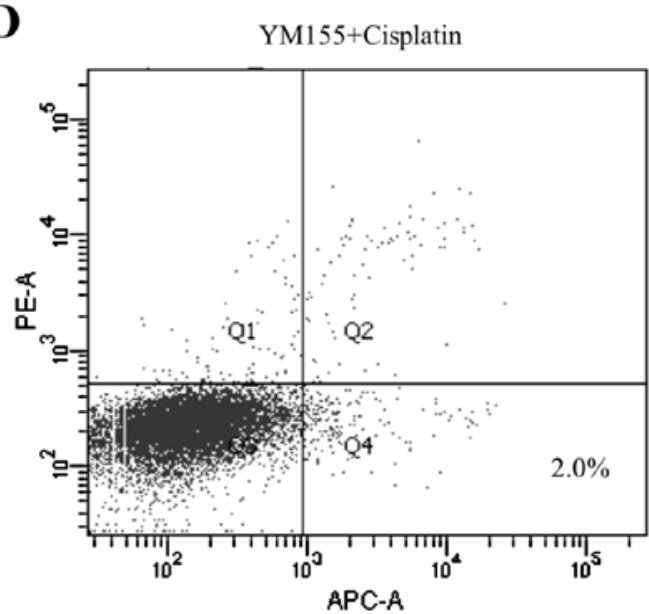

Figure 5. Flow cytometry using Annexin V and propidium iodide (PI). Apoptotic cells were defined as Annexin V-positive and PI-negative (Q4). The combination of YM155 and cisplatin (D) resulted in higher apoptosis than control (A) and either treatment alone (B and C).

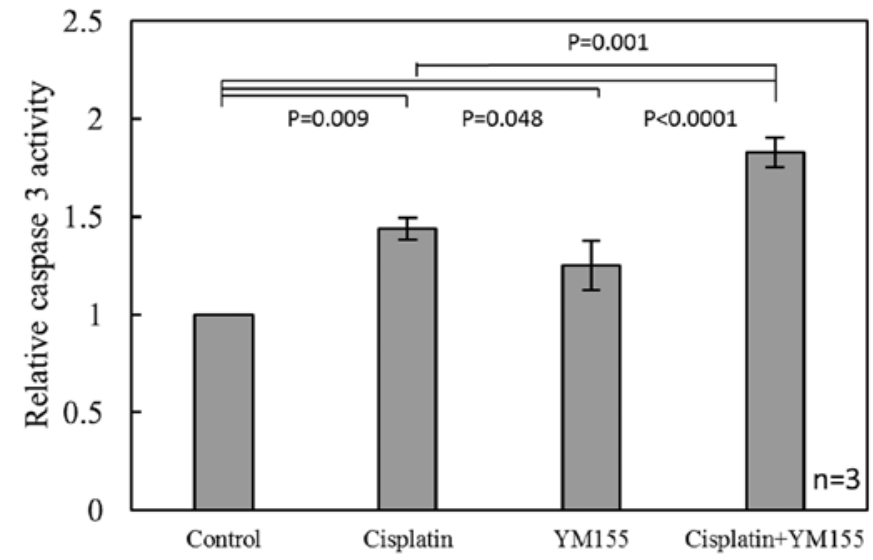

Figure 6. Relative caspase-3 activity following combined treatment with YM155 and cisplatin was greater than with either treatment alone.

tumors originated from RD cells, immunostaining verified that all specimens expressed the common human myogenic markers desmin and MyoD (Fig. 8A and B). To confirm survivin expression and apoptosis activity, survivin immunostaining and TUNEL staining were performed. Tumors treated with YM155 showed lower levels of survivin than control

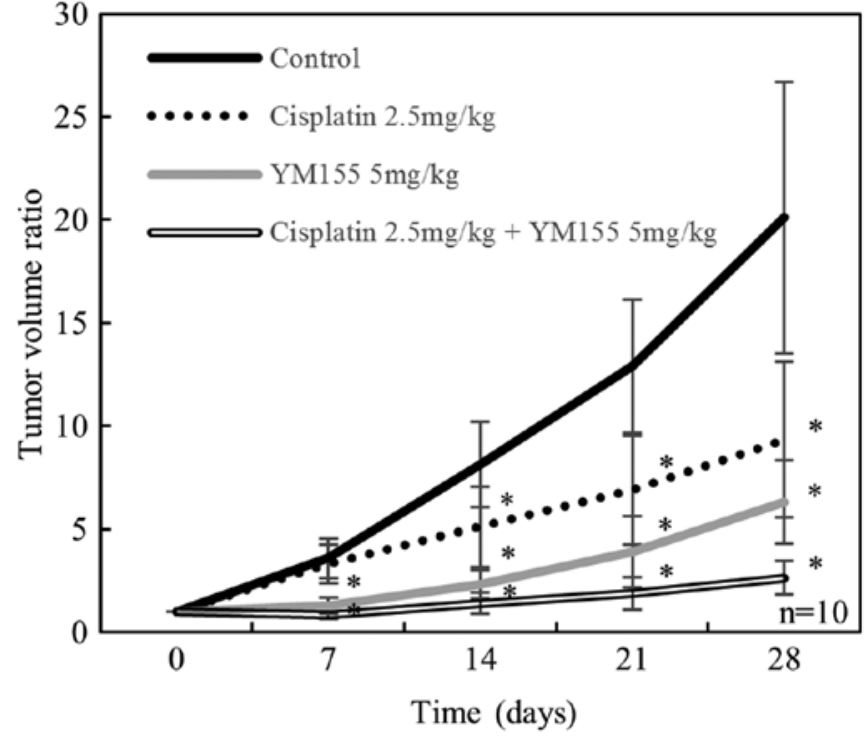

Figure 7. Nude mice (BALB/c nu/nu) subcutaneously injected with RD cells formed palpable tumors. Tumor volume was measured as described in Materials and methods, and the mean tumor volume over the 28-day experimental period was used to generate the graph. The growth rate was calculated during the treatment period. The combination of YM155 and cisplatin resulted in the greatest inhibition of tumor growth $\left({ }^{*} \mathrm{P}<0.0001\right)$. 
A

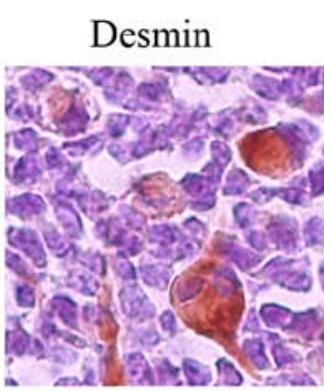

C

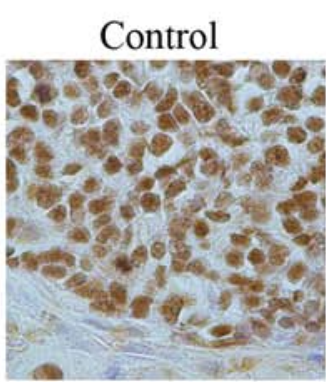

G

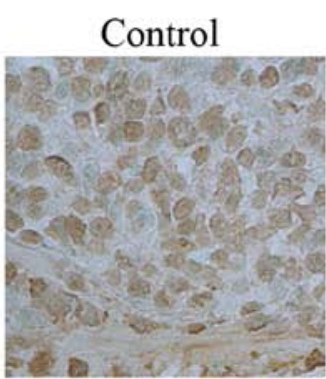

B

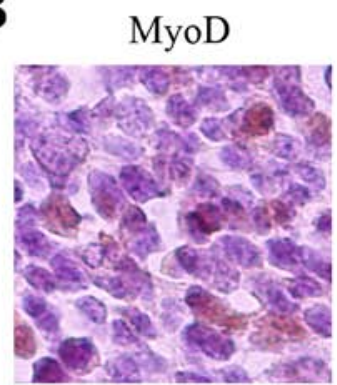

D

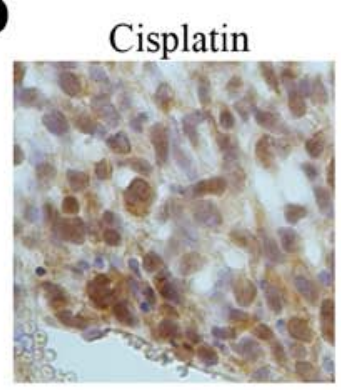

H

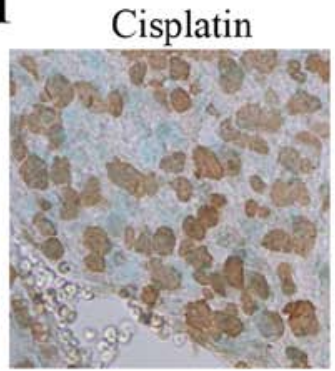

E

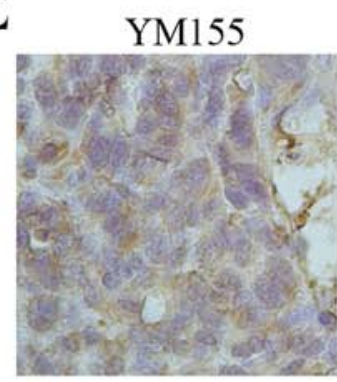

I

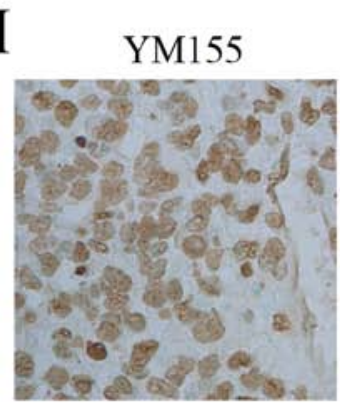

F YM155+ Cisplatin

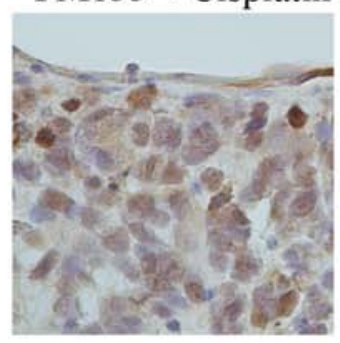

\section{$\mathbf{J}$}

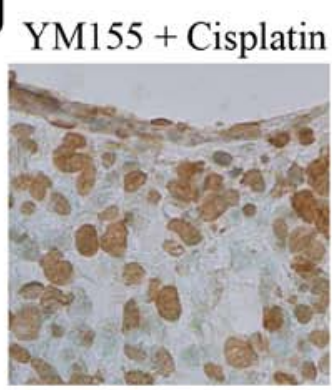

Figure 8. Histology of RMS xenografts. Myogenic markers desmin (A) and myoD (B) expressed in human RMS xenografts. Histology of RMS xenografts. Immunostaining with anti-survivin antibody (E and F). Histology of RMS xenografts. TUNEL staining (G-J).

untreated tumors (Fig. 8C-F). We observed an increase in TUNEL staining in YM155-treated tumors, consistent with the observed growth inhibition of treated tumors (Fig. 8G-J).

\section{Discussion}

The mainstay of chemotherapy for RMS is still the VAC regimen. Other drugs are used for second-line treatment and cisplatin has been added in higher risk patients, but to date there is no definitive evidence of improved outcomes with the addition of these drugs, despite evidence of response in the relapse setting (6). The estimated 5-year event-free survival rates in patients with low-, intermediate-, and high-risk disease are 95, 65 and 15\%, respectively (32). Therefore, a new therapeutic strategy is critically needed. Here, we examined the therapeutic potential of YM155 alone and in combination with cisplatin in RD cells in vitro and in a preclinical RD cell model. We found that YM155 exhibited significant antiproliferative activity and resulted in tumor regression in both $\mathrm{RD}$ cells and an established xenograft model.

Survivin is highly expressed in a broad range of solid tumors and hematological malignancies. Increased survivin expression in cancer patients is an unfavorable prognostic marker correlating with decreased overall survival in several malignancies, including lung $(33,34)$, gastric $(35)$, colorectal (18), and breast carcinomas (14), hepatocellular carcinoma (36) as well as in pediatric solid tumors such as neuroblastoma (37), and hepatoblastoma (23). Relationship between survivin expression and RMS are still unknown. However, some antiapoptotic activities were detected in RMS $(38,39)$. Survivin was strongly overexpressed in both alveolar and embryonal RMS cell lines and in RMS biopsy specimens (40). Blocking survivin expression by RNA interference reduces proliferation and viability of RMS xenotransplants (41). Downregulation of survivin in RMS cells increased their susceptibility toward a T-cell attack (40).

The success of anticancer therapies is often limited by the development of resistance to apoptosis, which may result from defects in common apoptotic pathways (42). In this context, approaches to counteract the action of survivin in tumor cells have been proposed with the dual aims of inhibiting tumor growth through promotion of spontaneous apoptosis and of enhancing the tumor cell response to apoptosis-inducing agents (11). In this study, we found that the combination of YM155 and the platinum compound cisplatin induced RD cell apoptosis as well as the activation of caspase- 3 to an extent greater than with either agent alone. Our findings thus suggest that YM155 acts in a synergistic manner to promote the induc- 
tion of apoptosis by cisplatin and possibly other platinum compounds.

Survivin was previously shown to enhance tumor cell survival after radiation exposure through regulation of doublestrand break repair (43). Iwasa et al showed that YM155 inhibited the repair of radiation-induced double-strand breaks in non-small cell lung cancer cells, and that this effect likely accounted for the observed radiosensitizing action of YM155 (44). They suggested that the observed chemosensitisation by YM155 is attributable to inhibition of the DNA damage repair induced by cisplatin (28). Further studies will be required to determine the mechanism underlying the effect of YM155 on DNA repair.

In our in vitro study, YM155 synergistically enhanced the antitumor activity of cisplatin in RD cells. In addition, YM155 combined with cisplatin resulted in greater tumor reduction than either treatment alone in an established xenograft model. Taken together, these findings suggest that YM155 may be a promising candidate for RMS therapy as a novel apoptosis inducer with survivin-suppressive activity. Further extensive research on survivin-mediated apoptosis defects may allow for the rational identification of RMS patients who would most likely respond to YM155. Combination chemotherapy is typically employed to achieve a better response rate than monotherapy, and is generally designed empirically using drugs that act through different cytotoxic mechanisms with less overlapping toxicity. Although cisplatin is a second-line drug for RMS, in combination with YM155 it may have a role in the therapy of VAC-resistant patients. Here, we found that YM155 plus cisplatin resulted in more intense apoptosis than either treatment alone. In addition, YM155 potentiated the antitumor activity of cisplatin without exacerbating weight loss in an established xenograft model.

In a toxicological study, short-term exposure to YM155 at high blood concentrations caused cardiotoxicity in the form of atrioventricular block and myocardial degeneration/necrosis, as well as nephrotoxicity, mainly displayed as proximal tubular necrosis and increased serum creatinine. No serious adverse hematological events related to drug treatment were reported in phase I studies of YM155 as single-agent therapy (45). A recent phase II study showed that YM155 monotherapy was safe but only moderately effective in patients with advanced lung cancer (46). Given the limited efficacy of YM155 as a single agent in clinical settings, the combination of YM155 with other agents may be beneficial. Furthermore, YM155 can reduce cisplatin dose in combination chemotherapy. It may enable to decrease serious side effect of cisplatin such as hearing loss, infertility and secondary malignancy. We have shown that the treatment of RD cells with YM155 resulted in a marked increase in the antitumor effects of cisplatin both in vitro and in vivo, suggesting that the combination of YM155 and platinum compounds may have potential as a novel therapeutic regimen. Clinical studies of YM155 in combination with platinum-based chemotherapies are thus warranted.

In conclusion, long-term chemotherapy is often limited by the development of drug resistance and cumulative side effects. This study showed that YM155 increased the sensitivity of cisplatin by suppressing survivin expression in the embryonal RMS cell line RD. The use of YM155 as an apoptosis inducer, either alone or in combination with cisplatin, may be warranted for the treatment of drug-resistant RMS.

\section{Acknowledgements}

The authors thank Miyoko Kawatsu for her assistance with our experiments.

\section{References}

1. Gurney J, Young JL Jr, Roffers SD, Smith MA and Bunin CR: Soft tissue sarcomas. In: Cancer Incidence and Survival Among Children and Adolescents: United States SEER Program 1975-1995. Ries LAG, Smith MA, Gurney JG, Linet M, Tamra T, Young JL and Bunin GR (eds). SEER Program, Benthesda, MD, 1999.

2. Flamant F, Rodary C, Rey A, Praquin MT, Sommelet D, Quintana E, Theobald S, Brunat-Mentigny M, Otten J, Voûte PA, et al: Treatment of non-metastatic rhabdomyosarcomas in childhood and adolescence. Results of the second study of the International Society of Paediatric Oncology: MMT84. Eur J Cancer 34: 1050-1062, 1998.

3. Crist W, Gehan EA, Ragab AH, Dickman PS, Donaldson SS, Fryer C, Hammond D, Hays DM, Herrmann J, Heyn R, et al: The Third Intergroup Rhabdomyosarcoma Study. J Clin Oncol 13: 610-630, 1995.

4. Donaldson SS: The value of adjuvant chemotherapy in the management of sarcomas in children. Cancer 55 (Suppl): 2184-2197, 1985.

5. Smith MA, Seibel NL, Altekruse SF, Ries LA, Melbert DL, O'Leary M, Smith FO and Reaman GH: Outcomes for children and adolescents with cancer: Challenges for the twenty-first century. J Clin Oncol 28: 2625-2634, 2010.

6. Lager JJ, Lyden ER, Anderson JR, Pappo AS, Meyer WH and Breitfeld PP; Soft Tissue Sarcoma Committee of the Children's Oncology Group: Pooled analysis of phase II window studies in children with contemporary high-risk metastatic rhabdomyosarcoma: A report from the Soft Tissue Sarcoma Committee of the Children's Oncology Group. J Clin Oncol 24: 3415-3422, 2006.

7. Carli M, Colombatti R, Oberlin O, Bisogno G, Treuner J, Koscielniak E, Tridello G, Garaventa A, Pinkerton R and Stevens M: European intergroup studies (MMT4-89 and MMT4-91) on childhood metastatic rhabdomyosarcoma: Final results and analysis of prognostic factors. J Clin Oncol 22: 4787-4794, 2004.

8. Breneman JC, Lyden E, Pappo AS, Link MP, Anderson JR, Parham DM, Qualman SJ, Wharam MD, Donaldson SS, Maurer HM, et al: Prognostic factors and clinical outcomes in children and adolescents with metastatic rhabdomyosarcoma - a report from the Intergroup Rhabdomyosarcoma Study IV. J Clin Oncol 21: 78-84, 2003.

9. Walterhouse DO, Pappo AS, Meza JL, Breneman JC, HayesJordan AA, Parham DM, Cripe TP, Anderson JR, Meyer WH and Hawkins DS: Shorter-duration therapy using vincristine, dactinomycin, and lower-dose cyclophosphamide with or without radiotherapy for patients with newly diagnosed low-risk rhabdomyosarcoma: A report from the Soft Tissue Sarcoma Committee of the Children's Oncology Group. J Clin Oncol 32: 3547-3552, 2014.

10. Weigel BJ, Lyden E, Anderson JR, Meyer WH, Parham DM, Rodeberg DA, Michalski JM, Hawkins DS and Arndt CA: Intensive multiagent therapy, including dose-compressed cycles of ifosfamide/etoposide and vincristine/doxorubicin/cyclophosphamide, irinotecan, and radiation, in patients with high-risk rhabdomyosarcoma: A Report From the Children's Oncology Group. J Clin Oncol 34: 117-122, 2016.

11. Altieri DC: Validating survivin as a cancer therapeutic target. Nat Rev Cancer 3: 46-54, 2003.

12. Tanaka C, Uzawa K, Shibahara T, Yokoe H, Noma H and Tanzawa H: Expression of an inhibitor of apoptosis, survivin, in oral carcinogenesis. J Dent Res 82: 607-611, 2003.

13. Reed JC: The Survivin saga goes in vivo. J Clin Invest 108: 965-969, 2001.

14. Ryan BM, Konecny GE, Kahlert S, Wang HJ, Untch M, Meng G, Pegram MD, Podratz KC, Crown J, Slamon DJ, et al: Survivin expression in breast cancer predicts clinical outcome and is associated with HER2, VEGF, urokinase plasminogen activator and PAI-1. Ann Oncol 17: 597-604, 2006. 
15. Rosato A, Pivetta M, Parenti A, Iaderosa GA, Zoso A, Milan G, Mandruzzato S, Del Bianco P, Ruol A, Zaninotto G, et al: Survivin in esophageal cancer: An accurate prognostic marker for squamous cell carcinoma but not adenocarcinoma. Int J Cancer 119: 1717-1722, 2006

16. Haberler C, Slave I, Czech T, Gelpi E, Heinzl H, Budka H, Urban C, Scarpatetti M, Ebetsberger-Dachs G, Schindler C, et al: Histopathological prognostic factors in medulloblastoma: High expression of survivin is related to unfavourable outcome. Eur J Cancer 42: 2996-3003, 2006.

17. Mori T, Doi R, Toyoda E, Koizumi M, Ito D, Kami K, Kida A, Masui T, Kawaguchi Y and Fujimoto K: Regulation of the resistance to TRAIL-induced apoptosis as a new strategy for pancreatic cancer. Surgery 138: 71-77, 2005.

18. Kawasaki H, Toyoda M, Shinohara H, Okuda J, Watanabe I, Yamamoto T, Tanaka K, Tenjo T and Tanigawa N: Expression of survivin correlates with apoptosis, proliferation, and angiogenesis during human colorectal tumorigenesis. Cancer 91: 2026-2032, 2001.

19. Kato J, Kuwabara Y, Mitani M, Shinoda N, Sato A, Toyama T, Mitsui A, Nishiwaki T, Moriyama S, Kudo J, et al: Expression of survivin in esophageal cancer: Correlation with the prognosis and response to chemotherapy. Int J Cancer 95: 92-95, 2001.

20. Grossman D, Kim PJ, Schechner JS and Altieri DC: Inhibition of melanoma tumor growth in vivo by survivin targeting. Proc Natl Acad Sci USA 98: 635-640, 2001.

21. Olie RA, Simões-Wüst AP, Baumann B, Leech SH, Fabbro D, Stahel RA and Zangemeister-Wittke U: A novel antisense oligonucleotide targeting survivin expression induces apoptosis and sensitizes lung cancer cells to chemotherapy. Cancer Res 60 : 2805-2809, 2000.

22. Yamamoto H, Ngan CY and Monden M: Cancer cells survive with survivin. Cancer Sci 99: 1709-1714, 2008.

23. Uehara S, Oue T, Kawatsu M, Nara K and Fukuzawa M: Increased expression of survivin in hepatoblastoma after chemotherapy. Eur J Pediatr Surg 23: 400-404, 2013.

24. Nakahara T, Kita A, Yamanaka K, Mori M, Amino N, Takeuchi M, Tominaga F, Hatakeyama S, Kinoyama I, Matsuhisa A, et al: YM155, a novel small-molecule survivin suppressant, induces regression of established human hormone-refractory prostate tumor xenografts. Cancer Res 67: 8014-8021, 2007.

25. Kita A, Nakahara T, Yamanaka K, Nakano K, Nakata M, Mori M, Kaneko N, Koutoku H, Izumisawa N and Sasamata M: Antitumor effects of YM155, a novel survivin suppressant, against human aggressive non-Hodgkin lymphoma. Leuk Res 35: 787-792, 2011.

26. Kumar B, Yadav A, Lang JC, Cipolla MJ, Schmitt AC, Arradaza N, Teknos TN and Kumar P: YM155 reverses cisplatin resistance in head and neck cancer by decreasing cytoplasmic survivin levels. Mol Cancer Ther 11: 1988-1998, 2012

27. Nakahara T, Yamanaka K, Hatakeyama S, Kita A, Takeuchi M, Kinoyama I, Matsuhisa A, Nakano K, Shishido T, Koutoku H, et al: YM155, a novel survivin suppressant, enhances taxaneinduced apoptosis and tumor regression in a human Calu 6 lung cancer xenograft model. Anticancer Drugs 22: 454-462, 2011.

28. Iwasa T, Okamoto I, Takezawa K, Yamanaka K, Nakahara T, Kita A, Koutoku H, Sasamata M, Hatashita E, Yamada Y, et al Marked anti-tumour activity of the combination of YM155, a novel survivin suppressant, and platinum-based drugs. Br J Cancer 103: 36-42, 2010

29. McAllister RM, Melnyk J, Finkelstein JZ, Adams EC Jr and Gardner MB: Cultivation in vitro of cells derived from a human rhabdomyosarcoma. Cancer 24: 520-526, 1969.

30. Mir R, Stanzani E, Martinez-Soler F, Villanueva A, Vidal A, Condom E, Ponce J, Gil J, Tortosa A and Giménez-Bonafé P: YM155 sensitizes ovarian cancer cells to cisplatin inducing apoptosis and tumor regression. Gynecol Oncol 132: 211-220, 2014.
31. Liang H, Zhang L, Xu R and Ju XL: Silencing of survivin using YM155 induces apoptosis and chemosensitization in neuroblastomas cells. Eur Rev Med Pharmacol Sci 17: 2909-2915, 2013.

32. Hawkins DS, Spunt SL and Skapek SX; COG Soft Tissue Sarcoma Committee: Children's Oncology Group's 2013 blueprint for research: Soft tissue sarcomas. Pediatr Blood Cancer 60: 1001-1008, 2013.

33. Zhang K, Li Y, Liu W, Gao X and Zhang K: Silencing survivin expression inhibits the tumor growth of non-small-cell lung cancer cells in vitro and in vivo. Mol Med Rep 11: 639-644, 2015.

34. Dai CH, Li J, Shi SB, Yu LC, Ge LP and Chen P: Survivin and Smac gene expressions but not livin are predictors of prognosis in non-small cell lung cancer patients treated with adjuvant chemotherapy following surgery. Jpn J Clin Oncol 40: 327-335, 2010.

35. Vallböhmer D, Drebber U, Schneider PM, Baldus S, Bollschweiler E, Brabender J, Warnecke-Eberz U, Mönig S, Hölscher AH and Metzger R: Survivin expression in gastric cancer: Association with histomorphological response to neoadjuvant therapy and prognosis. J Surg Oncol 99: 409-413, 2009.

36. Augello C, Caruso L, Maggioni M, Donadon M, Montorsi M, Santambrogio R, Torzilli G, Vaira V, Pellegrini C, Roncalli M, et al: Inhibitors of apoptosis proteins (IAPs) expression and their prognostic significance in hepatocellular carcinoma. BMC Cancer 9: 125, 2009.

37. Islam A, Kageyama H, Takada N, Kawamoto T, Takayasu H, Isogai E, Ohira M, Hashizume K, Kobayashi H, Kaneko Y, et al: High expression of Survivin, mapped to $17 \mathrm{q} 25$, is significantly associated with poor prognostic factors and promotes cell survival in human neuroblastoma. Oncogene 19: 617-623, 2000.

38. Wachtel M and Schäfer BW: Targets for cancer therapy in childhood sarcomas. Cancer Treat Rev 36: 318-327, 2010.

39. Fulda S: Apoptosis pathways and their therapeutic exploitation in pancreatic cancer. J Cell Mol Med 13: 1221-1227, 2009.

40. Simon-Keller K, Paschen A, Hombach AA, Ströbel P, Coindre JM, Eichmüller SB, Vincent A, Gattenlöhner S, Hoppe F, Leuschner I, et al: Survivin blockade sensitizes rhabdomyosarcoma cells for lysis by fetal acetylcholine receptor-redirected T cells. Am J Pathol 182: 2121-2131, 2013.

41. Caldas H, Holloway MP, Hall BM, Qualman SJ and Altura RA: Survivin-directed RNA interference cocktail is a potent suppressor of tumour growth in vivo. J Med Genet 43: 119-128, 2006.

42. Hanahan D and Weinberg RA: The hallmarks of cancer. Cell 100: 57-70, 2000 .

43. Chakravarti A, Zhai GG, Zhang M, Malhotra R, Latham DE, Delaney MA, Robe P, Nestler U, Song Q and Loeffler J: Survivin enhances radiation resistance in primary human glioblastoma cells via caspase-independent mechanisms. Oncogene 23 : 7494-7506, 2004.

44. Iwasa T, Okamoto I, Suzuki M, Nakahara T, Yamanaka K, Hatashita E, Yamada Y, Fukuoka M, Ono K and Nakagawa K: Radiosensitizing effect of YM155, a novel small-molecule survivin suppressant, in non-small cell lung cancer cell lines. Clin Cancer Res 14: 6496-6504, 2008.

45. Tolcher AW, Mita A, Lewis LD, Garrett CR, Till E, Daud AI, Patnaik A, Papadopoulos K, Takimoto C, Bartels P, et al: Phase I and pharmacokinetic study of YM155, a small-molecule inhibitor of survivin. J Clin Oncol 26: 5198-5203, 2008.

46. Giaccone G, Zatloukal P, Roubec J, Floor K, Musil J, Kuta M, van Klaveren RJ, Chaudhary S, Gunther A and Shamsili S: Multicenter phase II trial of YM155, a small-molecule suppressor of survivin, in patients with advanced, refractory, non-small-cell lung cancer. J Clin Oncol 27: 4481-4486, 2009. 